\title{
GEOPOLITICA E DIPLOMAZIA. IL CASO ITALIA BRASILE
}

\author{
GEOPOLÍTICA E DIPLOMACIA. O CASO ITÁLIA BRASIL
}

GEOPOLITICS AND DIPLOMACY. THE CASE ITALY BRAZIL

\author{
Aniello Angelo Avella \\ Università di Roma Tor Vergata \\ Universidade do Estado do Rio de Janeiro \\ nelloavella@ig.com.br
}

Sintesi:

Il Brasile, dove risiede la più grande comunità di oriundi italiani al mondo, è uno dei principali partners dell'Italia nel panorama politico internazionale, non solo per le sue grandi risorse naturali e il notevole sviluppo economico ma soprattutto per l'affinità e la reciproca simpatia fra i due popoli. In questi ultimi anni la diplomazia ha modificato sensibilmente le sue strategie rispetto al passato: oggi vengono curati soprattutto gli aspetti antropologico-culturali, si promuove la cooperazione fra università e centri di ricerca, si incentiva l'innovazione e l'internazionalizzazione. Il "Momento Italia Brasile", con più di 400 eventi realizzati in tutti gli Stati della Federazione, ha messo in evidenza le profonde connessioni e le eccellenti possibilità di cooperazione bilaterale, sulla base della comune eredità latina.

Parole-chiave: Italia, Brasile, Geopolitica, Diplomazia, Eredità Culturale.

\section{Resumo:}

O Brasil, País que abriga a maior comunidade de"oriundi" no mundo, é um dos principais partners da Itália no panorama político internacional, não apenas por seus grandes recursos naturais e seu notável desenvolvimento econômico, mas especialmente pela afinidade e a simpatia recíproca entre os dois povos. Nos últimos anos, a diplomacia mudou sensivelmente suas estratégias em relação ao passado: atualmente preocupa-se em particularcom os aspetos antropológicoculturais, promove a cooperação entre universidades e centros de pesquisa, estimula a inovação e a internacionalização. A manifestação, intitulada“"Momento Itália Brasil”, com mais de 400 eventos realizados, evidenciou as profundas conexões e as excelentes possibilidades de cooperação bilateral,com base na comum herança latina.

Palavras-Chave: Itália, Brasil, Geopolítica, Diplomacia, Herança Cultural. 


\begin{abstract}
:
Brazil, home to the largest community ofItalian originin the world,isone of the main partnersof Italy in theinternational political landscape, not only for its greatnatural resources and theconsiderable economic developmentbut also forthe affinityand mutual sympathy betweenthe two peoples..In recent years, diplomacy haschanged itsstrategies compared to the past: now, especiallytaking care ofanthropologicaland culturalaspects, it promotes cooperation between universitiesand research centers, it stimulates innovation and internationalization now, especiallytaking care ofanthropologicaland culturalaspects, it promotes cooperation between universitiesand research centers, it stimulates innovation and internationalization. "Momento Italia Brasile", with more than 400events heldin all theStates of the Federation, highlightedthe deepconnections andexcellent opportunities forbilateral cooperationon the basis of commonLatinheritage.
\end{abstract}

Key-words: Italy, Brazil, Geopolitics, Diplomacy, Cultural Heritage.

Le celebrazioni del centocinquantesimo anniversario dell'unità d’Italia hanno avuto un ideale prosecuzione negli eventi del "Momento Italia Brasile", realizzati nel Paese sudamericano fra ottobre 2011 e luglio 2012. Progettata e organizzata dalla nostra ambasciata a Brasília col sostegno degli Istituti di Cultura di Rio de Janeiro e São Paulo, la manifestazione è stata una rassegna degli aspetti più peculiari della realtà italiana, presentati al pubbliconell'intento di valorizzare i forti vincoli da sempre esistenti fra i due popoli e favorire lo sviluppo di attività di cooperazione nei più svariati campi della vita sociale, economica, artistica.

Le numerose iniziative realizzate hanno evidenziato un elemento di cui l'opinione pubblica del nostro Paese non sembra avere sufficiente consapevolezza: la straordinaria presenza di oriundi italiani in Brasile, enorme serbatoio di valori comuni e potenzialità di ogni tipo. Dati ufficiosi ma attendibili, diffusi dalle rappresentanze diplomatiche, riferiscono di circa trenta milioni di persone, che costituiscono la più grande comunità al mondo di italo-discendenti.

Nella cultura italiana si è sedimentata nel corso dei secoli una visione deformata del mondo iberico: concentrando la propria attenzione quasi esclusivamente sulla Spagna, anche per le vicende storiche che è superfluo ricordare in questa sede, gli intellettuali e gli osservatori - fino a non molto tempo fa- hanno guardato con limitato interesse al mondo lusitano nella sua componente europea e nelle sue propaggini oltremarine. Cosicché anche il Brasile si è andato connotando nel segno di una alterità vista soprattutto nei suoi aspetti Geo UERJ - Ano 14, no. 23,v. 2, $2^{\circ}$ semestre de 2012 p. 375-388

ISSN: 1415-7543 E-ISSN: 1981-9021

http://www.e-publicacoes.uerj.br/index.php/geouerj 
esotici e non ne sono stati approfonditi i complessi aspetti antropologici, insieme alle connessioni con la nostra tradizione e la nostra storia. Solo in tempi recenti si è instaurato un clima di maggiore apertura, anche in seguito alla crisi prodotta dalla globalizzazione, con la conseguente necessità di allargare gli orizzonti per riposizionarsi nei nuovi profili della geopolitica mondiale.Si rivelafondamentale, in questo contesto, il ruolo della diplomazia, alla quale sia 1'Italia che il Brasile hanno affidato il compito di favorire il dialogo attraverso la cooperazione scientifica e gli scambi culturali.

$\infty$

I rapporti bilaterali risalgono, come è noto, ai primordi della colonizzazioneportoghese e affondano le loro radici nella comune eredità latina. Diceva infatti Darcy Ribeiro che "Roma, incarnata in lusitanità, nelle Americhe si è vestita di carne india e di carne negra" e dunque "la nuova Roma sta qui", in America Latina, in Brasile (RIBEIROapud AVELLA, 2006, p. 53).

Già Sérgio Buarque de Holanda, professore all'università di Roma e presso i servizi culturali dell'ambasciata brasiliana in Italia agli inizi degli anni Cinquanta del '900, aveva messo in forte evidenza 1 " «Apporto italiano nella formazione del Brasile», titolo di un suo saggio pubblicato nel numero speciale da lui curato per la rivista senese Ausonia. Esiste fra i due popoli, aveva scritto, "un'affinità essenziale e ineluttabile" (BUARQUE DE HOLANDA, 2002,p.108). All'interno del saggio, egli aveva sottolineato con speciale enfasi l'importanza dell'Arcadia Romana e la sua influenza decisiva nella formazione di una cultura brasiliana consapevole della propria identità e differenza rispetto alla metropoli portoghese.

È noto, infatti, che le Accademie letterarie fondate nella seconda metà del Settecento a Minas Gerais e in altre zone della colonia s śispiravano più o meno direttamente al modello arcadico; numerosi intellettuali risultavano iscritti all'Arcadia romana, come il poeta epico José Basílio da Gama, che aveva assunto il nome di Termindo Serpilio. Non pochi di loroparteciparono almovimento indipendentista della "Inconfidência Mineira". Il poeta arcade Silva Alvarenga fu tra i promotori di una cospirazione organizzata e scoperta a Rio de Janeiro qualche anno dopo. Da notare, infine, che il cosiddetto "Patriarca dell'Indipendenza del Brasile”, José Bonifácio de Andrade, faceva parte dell'Arcadia col 
nome di Américo Elísio. Oltre che poeta, egli fu un insigne scenziato; la sua formazione scientifica avvenne a Coimbra, in un momento storico di generale fervore riformistico,sotto il magistero del naturalista Domenico Vandelli, nato a Padova nel 1736 e là laureatosi in medicina, chiamato poi in Portogallo dal Marchese di Pombal per la grande fama di cui godeva. Morto a Lisbona nel 1816, Vandelli è considerato una della maggiori autorità nel campo delle scienze naturali dell'Europa del tempo. Fra le sue innumerevoli pubblicazioni, di particolare rilievo è il Florae Lusitanicae etBrasiliensis Specimen (Coimbra, 1788).

La trama dei rapporti fra Italia, Portogallo e Brasile diventò ancora più fitta in seguito al matrimonio del figlio di Domenico Vandelli, Antonio Alessandro, con la figlia di José Bonifácio, Carlota Emília de Andrade (1819). Trasferitosi in Brasile con sua moglie, Antonio Alessandro Vandelli fu professore di Botanica e Scienze Naturali di D. Pedro II e della famiglia imperiale dal 1839 al 1862 (MARQUES, 2010).

$\infty$

Vinícius de Morais, diplomatico di professione, poeta e cantautore per passione, sosteneva che la letteratura e la musica sono i migliori ambasciatori del Brasile nel mondo. Non sono pochi i nomi di importanti figure della cultura brasiliana provenienti dal mondo della diplomazia; basti pensare a João Guimarães Rosa o a João Cabral de Melo Neto.

La funzione della cultura nell'ambito delle relazioni italo-brasiliane è notevolmente aumentata nel corso degli ultimi anni, caratterizzati sempre più dallo sviluppo della cosiddetta "società della conoscenza".

Tale processo ha influenzatol'approccio della diplomazia alle problematiche delle rispettive azioni di rappresentanza nei due Paesi. Lo dimostra, fra l'altro, il notevole contributo dato dalle ambasciate di Roma e Brasília all'allargamento dei rapporti di cooperazione scientifica e didatticainteruniversitaria.

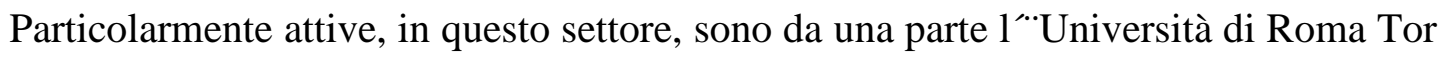
Vergata" e dall'altra la "Universidade do Estado do Rio de Janeiro", impegnate da anni in numerosi progetti di reciproco interesse, con scambio di docenti e discenti.

Sotto l'egida dell'ambasciata a Brasília, e in ragione dell'importanza strategica della loro collaborazione, i due atenei sono stati inseriti fra i protagonisti del "Momento Italia Brasile“ed hanno dato vita a una fitta serie di eventi. 
Dalla “Jornada Acadêmica“, realizzata presso l'università carioca nel novembre 2011, sono scaturiti programmi in vari campi della ricerca, in particolare della geografia e del turismo culturale, con la stipula di una convenzione con la "Società Geografica Italiana", rappresentata dal responsabile delle relazioni internazionali, Alfonso Giordano, a sua volta docente incaricato all'università di Tor Vergata.

La "Società Geografica Italiana" è una delle istituzioni più prestigiose del nostro Paese; fondata nel 1867, poco dopo l'unificazione, il suo primo statuto "elencava tra i fini sociali la conoscenza del territorio italiano, il sostegno ai viaggi di esplorazione e la promozione degli interessi economici italiani”(CERRETI, 2000, p. 12).

Opportunamente l'ambasciata d'Italia a Brasília ha favorito, attraverso l'Istituto di Cultura di Rio de Janeiro, la firma di un accordo di cooperazione fra la "Società Geografica italiana“ e 1“"Instituto Histórico e Geográfico Brasileiro“, nel rispetto della filosofia alla quale si è ispirato il "Momento Italia Brasile": mostrare il meglio delle eccellenze italiane per metterle in contatto coi possibilipartners brasiliani, onde stimolare azioni comuni rivolte all'internazionalizzazione e all'innovazione.

L’accordo fra "Società Geografica Italiana" e "Instituto Histórico e Geográfico Brasileiro" è stato sancito ufficialmente nel corso del "Seminário Brasil itália", tenutosi a Rio de Janeiro a marzo 2012. Il "Seminário" ha riunito nella sede dell'IHGB alcuni fra i maggiori studiosi dei due Paesi, che si sono confrontati sui principali aspetti della presenza e influenza italiana nella società brasiliana. I presidenti delle due istituzioni, Franco Salvatori (professore ordinario all’Università di Roma Tor Vergata) e Arno Wehling, hanno sottolineato il valore simbolico dell'atto col quale l'antica istituzione italiana si collega con la consorella d'oltre oceano, nata nel 1838 sotto gli auspici di D. Pedro II, la cui Revista continua a essere pubblicata regolarmentedalla fondazione con cadenza trimestrale; il suo motto è: "Hoc facit, ut longos durent bene gesta per annos. Et possit sera posteritate frui". Anche 1““Instituto Histórico e Geográfico Brasileiro“, come la "Società Geografica Italiana", agisce per promuovere il progresso delle scienze in nome dell'utile e del bene. 
O Filho da Serpente Encantada. Fotografias de Ermanno Stradelli: èil titolodi una esposizione inaugurata nei locali della "Universidade do Estado do Rio de Janeiro" a maggio 2012 e poi trasferita nei maggiori centri dello Stato di Rio de Janeiro, in attesa di essere portata in varie città del Brasile e concludere il suo itinerario a Manaus, dove il grande esploratore, geografo, etnologo, fotografo italiano morí nel 1926.

Stradelli, nato a Borgo Val di Taro nel 1852, è stato un vero e proprio ponte fra le due culture. Le sue relazioni dei viaggi effettuati nella regione amazzonica, pubblicate nel Bollettino della Società Geografica Italiana, di cui era socio, mostrano sincero interesse per le culture indigene e la loro difesa nei confronti di commercianti, militari, evangelizzatori bianchi, supposti portatori di civiltà. La sua opera piùampia, "Vocabulários da língua geral portuguez-nheéngatú e nheéngatú-portuguez", fu pubblicatapostumanel 1929 nella Revista do Instituto Histórico e Geográfico Brasileiro.

La mostra presenta per la prima volta al pubblico brasiliano una raccolta di fotografie rarissime, scattate da Stradelli in Amazzonia. Le foto sono di proprietà della "Società Geografica Italiana“, l'organizzazione è stata curata dall'Instituto de Geografia e dal Departamento Cultural della "U.E.R.J.", col patrocinio dell'ambasciata d'Italia e la cooperazione dei due istituti Italiani di Cultura di Rio e São Paulo.

L'evento dedicato a Stradelli è stato realizzato nell’ambito del "Momento Italia Brasile“, al pari dell'altra mostra intitolata Além de Pompeia. Redescobrindo os encantos de Stabiae, anch’essaospitataneilocalidella “Universidade do Estado do Rio de Janeiro”.

È noto che la vicenda di Pompei, la sua distruzione sotto la lava del vulcano, s’è trasformata in una delle figure di maggiore impattosull'immaginario collettivouniversale, alimentata da cinema, letteratura, arti plastiche ecc. Solo Ercolano, colpita dalla stessa calamità, riesce a conservare un suo spazio nella memoria accanto all'altra più famosa città vesuviana. Meno nota è invece Stabiae, "perché scavata in epoca borbonica (1749-1762 e 1775-1782) e poi nuovamente sepolta. Per tutto il XIX secolo non si ebbero ricerche specifiche rivolte alla conoscenza di Stabiae. L'attenzione e le risorse erano tutte rivolte a Pompei“ (JACOBELLI, 2012, p. 15).

La mostra ospitata dalla "Universidade do Estado do Rio de Janeiro" contribuisce a dare una visione più ampia e articolata della vita quotidiana all'epoca romana. Se infatti Pompei si caratterizzava per la sua ricchezza derivante dall'industria e dal commercio, ed 
Ercolano per l'eleganza grecizzante dovuta alla prossimità con Neapolis, Stabiae si distingueva per il lusso delle villae d'otium destinate alla villeggiatura delle classi alte.

L’iniziativa si collega direttamente alle attività archeologice dell’imperatrice napoletana del Brasile, Teresa Cristina di Borbone (1822-1889). Moglie di D. Pedro II, la sua immagine è stata offuscata da quella del consorte, esaltato come "Imperatore Filosofo", grande intellettuale nei cui confronti Teresa Cristina sarebbe solo una pallida ombra, priva di spessore culturale. Un recentissimo libro ne evidenzia invece il notevole apporto nello sviluppo delle relazioni fra Italia e Brasile durante il XIX secolo, quando si posero le basi dei flussi migratori che diedero origine all'attuale comunità di oriundi. Nell'azione di Teresa Cristina l'archeologia fu molto importante, come testimoniano le collezioni di reperti italici, etruschi e pompeiani oggi esposte nelle sale del “Museu Nacional"(AVELLA 2012).

Collocata a poche centinaia di metri dalla "Quinta da Boa Vista“, la residenza privata degli imperatori poi diventata museo, l'esposizioneAlém de Pompeia. Redescobrindo os encantos de Stabiae si può considerare come il complemento ideale della collezione creata a Rio da Teresa Cristina di Borbone, della quale nel 2012 ricorreva il centonaventesimo anniversario della nascita. La mostra riveste, oltre che notevole valore simbolico e scientifico, un significato strategico di grande portata ai fini delle relazioni italo-brasiliane; per queste ragioni sia l'ambasciata a Brasília sia quella a Roma hanno appoggiato e patrocinato l'iniziativa della fondazione campana "Restoring Ancient Stabiae", organizzatrice della mostra insieme alla "Soprintendenza Speciale per i Beni Archeologici di Napoli e Pompei“e al rettorato della "Universidade do Estado do Rio de Janeiro".

Per decisione dell'ambasciatore Gherardo La Francesca, l'apertura ufficiale del "Momento Italia Brasile" è avvenuta a Rio de Janeiro, considerando che la "Città Meravigliosa“ non è solo la cartolina postale del Paese ma anche un grande polo di attività culturali, sportive, imprenditoriali.

In una piovosa serata di metà ottobre del 2011, un centinaio di artisti italiani e brasiliani si sono esibiti per quasi due ore nella piazza Floriano, cuore della Cinelândia, 
tradizionale luogo di incontro della megalopoli carioca. Il grandioso spettacolo concepito da Valerio Festi s'intitolava Ensaio sobre a Beleza; palchi mobili e gigantesche macchine volanti attraversavano la piazza, le facciate del "Theatro Municipal"e della "Camâra dos Vereadores" si mischiavano alle immagini che venivano proiettate, coinvolgendo le oltre 5.000 persone del pubblico in un gioco ispirato al principio barocco esplicitamente asssunto dall'ideatore dell'evento: “il fin, la meraviglia”. Fra allusioni, citazioni, metafore, allegorie di vario tipo, il saggio sviluppava un ideale itinerario dal Rinascimento italiano fino all'arte contemporanea; all'inizio, una bambina seduta accanto a suo nonno domanda perché gli piace tanto andare al teatro. Egli risponde che così si ricorda della gioventù, di quando è arrivato in Brasile dall'Italia, e dice che la contemplazione della musica e della bellezza rappresenta il vero legame fra i due Paesi.

Fra i circa 400 eventi del "Momento Italia Brasile", la cultura e l'arte hanno svolto un ruolo di primo piano. Le mostre su Roma imperiale, Vasari, Caravaggio, De Chirico, Modigliani, hanno mobilitato moltitudini di visitatori in tutto il Paese; il restauro della "Casa Rosada“a Belém do Pará, edificio del secolo XVIII attribuito all'architetto bolognese Antonio Landi, ha esaltato il senso di integrazione e cooperazione fra le due culture. Le feste popolari come quelle dell'uva e della polenta, i concerti nelle piazze e nei teatri, la rassegna "Brasil Sabor", con centinaia di ristoranti che offrivano gastronomia italiana, hanno mostrato quanto siano amate e conservate le tradizioni nel "Brasile Italiano". Nel suo cuore più profondo, l'Amazzonia, un battello ha portato il nostro cinema fino ai villaggi più sperduti. Lo stile della moda italiana è stato ammirato in decine di sfilate nei luoghi deputati al fashion.

Durante la "Conferenza Internazionale sullo Sviluppo Sostenibile Rio + 20", la rappresentanza diplomatica a Brasília ha presentato i frutti del "Progetto Ambasciata Verde", esempio notevole di cooperazione tecnologica bilaterale. A partire dal 2010, infatti, la sede s'è andata trasformando in laboratorio di esperienze eco-sostenibili. Sul tetto sono stati installati 405 pannelli fotovoltaici che producono energia elettrica per l'uso degli uffici, delle abitazioni degli addetti alla sicurezza, della residenza dell'ambasciatore, riducendo drasticamente le emissioni di carbonio. L'impianto idrico è stato rinnovato adottando un sistema di filtri naturali e la depurazione si conclude attraverso l'azione delle piante, che trasformano le acque sporche in acque potabili. Esse vengono riutilizzate per l'irrigazione dei giardini. Partners del progetto sono stati l'impresa italiana "Enel Green Power" e le 
autorità brasiliane competenti in materia: "Agência Nacional de Energia Elétrica", "Companhia Energética de Brasília“, "Companhia de Saneamento Ambiental do Distrito Federal".

In Brasile operano più di 700 imprese italiane di grandi, medie e piccole dimensioni, spesso in regime di joint venturecon aziende locali. Esse danno occupazione a circa 130.000 persone e, nell'indotto, a quasi 500.000. I settori più attivi sono quelli del marmo, della nautica, dell'automotivo, con importanti prospettive di crescita anche in altri campi. Per favorire tale processo, le due ambasciate si sono impegnate nel promuovere iniziative come conferenze, seminari, missioni rivolte all'internazionalizzazione attraverso strategie di crescita e sviluppo sostenibile. Il documento pubblicato a cura dell’ambasciata di Brasília col titolo Contribuição Italiana para o Desenvolvimento Econômico do Brasil (2012) è un utile strumento conoscitivo della realtà attuale e delle potenzialità future; disegna una mappa dettagliata delle imprese operanti sul territorio, che hanno fatto registrare negli ultimi dieci anni un incremento degli investimenti italiani in Brasile pari al 220\%.

Gli aspetti economici delle relazioni bilaterali appaiono oggi ispirati a una visione nuova, assai più attenta alle tematiche sociali e alle ricadute sulla vita delle persone rispetto a quanto avveniva in passato. Se la globalizzazione ha alterato profondamente antichi equilibri di potere, con effetti spesso devastanti per gruppi e soggetti meno preparati ad affrontarne le sfide, essa ha messo in luce l'importanza della solidarietà e del recupero dei grandi capitali rappresentati dalla comunanza di valori e tradizioni.

Italia e Brasile dimostrano di aver compreso le esigenze dei tempi nuovi, dando spazio gradatamente maggiore a una dimensione "umanistica“dei loro rapporti.Ne è un esempio la realizzazione della Guida alle fonti della Storia del Brasile in Italia/Guia para as Fontes da História do Brasil na Itália, che riunisce e classifica organicamente l'enorme quantità di documenti relativi al Brasile coloniale depositati in biblioteche e archivi italiani. L'iniziativa fa parte del progetto "Resgate -Barão do Rio Branco", promosso dal "Ministério da Cultura“ brasiliano; il lavoro è stato eseguito da una équipe di ricercatori dei due Paesi, col coordinamento scientifico dell“'Università di Roma Tor Vergata“, l'opera in due volumi (versione italiana e portoghese) è curata dalla casa editrice della "Universidade do Estado do Rio de Janeiro“, a ulteriore dimostrazione dei vincoli esistenti fra queste istituzioni. 
L'operazione "Resgate" è stata sostenuta dal punto di vista logistico dall'ambasciata del Brasile a Roma; sull’altra sponda, l'ambasciata d’Italia a Brasília si è prodigata per favorire la circolazione degli studiosi e i contributi scientifici. Entrambi gli ambasciatori hanno voluto sottolineare il valore dell'opera scrivendo testi di presentazione.

Bisogna ricordare inoltre che il sistema universitario italiano è stato scelto come uno dei partners del Brasile nel progetto "Ciência sem Fronteiras", che prevede la concessione di circa 80.000 borse di studio; 6.000 studenti brasiliani nei prossimi 4 anni saranno accolti in Italia. Anche in questo caso, la spinta all'internazionalizzazione eall'innovazione si sposano con l'integrazione fra i due popoli.

$\infty$

Se è vero che grandi numeri caratterizzano i rapporti fra Italia e Brasile in tutti i comparti della vita sociale ed economica, esiste però una realtà difficile da esprimere in cifre. Come ha scritto l'ambasciatore La Francesca, "si tratta dell'affinità, simpatia e stima reciproca e della facilità che italiani e brasiliani hanno a lavorare e, perché no, a divertirsi insieme“ (LA FRANCESCA, 2012, p.3).

A partire da tale constatazione,la diplomazia odiernaattua strategie rivolte a favorire politiche capaci di produrre trasformazioni positive nella vita dei cittadini, considerando non solo le dinamiche economiche ma, prioritariamente, i diritti al benessere, alla salute, alla salvaguardia dell’ambiente. Ribaltando i principi della geopolitica classica, le relazionibilateralisi richiamanoal nesso dialettico fra due triadi interpretative: poterepolitica-strategia e geografia-ambiente-territorio; è in tale prospettiva che è stato creato il "Gruppo di Cooperazione Parlamentare Italo-Brasiliano", col decisivo apporto delle rappresentanze diplomatiche.

Grazie a questa nuova strategia è stato possibile superare, recentemente, alcuni momenti di difficoltà eincomprensioni in certi casi anche molto serie,nell'alveo della tradizionale amicizia e affinità di cui parlava l’ambasciatore La Francesca.

Qualcosa di simile era avvenuto anche nel secondo dopoguerra: dopo che Italia e Brasile avevano combattuto su fronti contrapposti, furono attivati canali diplomatici ufficiali e non. Si decise di affidare la ripresa del dialogo soprattutto alla mediazione di illustri personaggi, che fecero appello al comune patrimonio di cultura e valori spirituali.

Geo UERJ - Ano 14, no. 23,v. 2, $2^{\circ}$ semestre de 2012 p. 375-388

ISSN: 1415-7543 E-ISSN: 1981-9021

http://www.e-publicacoes.uerj.br/index.php/geouerj 
Nel 1946 giunsero in Brasile, su invito del magnate Assis Chateaubriand, Lina Bo e suo marito Pietro Maria Bardi, che fondarono il "Museu de Arte de São Paulo“; dopo la firma della "Dichiarazione di Amicizia e Cooperazione", nel 1949, fu riattivato il "Centro Cultural Brasil-Itália“ con a Rio de Janeiro, presieduto dallo storico Pedro Calmon e con un comitato direttivo di alto profilo scientifico Nel 1948 l'ingegnere Franco Zampari, napoletano di nascita, aveva fondato a São Paulo il "Teatro Brasileiro de Comédia", attribuendone la direzione ad Adolfo Celi. Poco dopo arrivarono Ruggero Jacobbi, Flaminio Bollini, Luciano Salce.

L’anno successivo lo stesso Zampari creò la prima compagnia brasiliana di produzione cinematografica, la "Vera Cruz", che nel 1950 produsse il film Caiçara per la regia di Adolfo Celi, un'opera chiaramente influenzata dal neo-realismo italiano.

Nel 1951 1'istituto “Angelicum“di Milano portò in Brasile la sua orchestra e una grande mostra di arte sacra, mentre 1“"Instituto Cultural Ítalo-Brasileiro“di São Paulo bandì un concorso per la migliore monografia sui rapporti fra i due Paesi.

Sull'altra sponda dell'oceano, l’ambasciata del Brasile a Roma iniziò una campagna per la diffusione della cultura e letteratura brasiliana; premi ed incentivi all'approfondimento della conoscenza mutua furono offerti da organismi pubblici e privati, fra cui la fondazione "Amerigo Rotellini”.

Nel 1953 venne creata la cattedra di “Studi Brasiliani”all’università di Roma, affidata a Sérgio Buarque de Holanda, all’epoca direttore del "Museu Paulista”, e in seguito al poeta Murilo Mendes.

L“A Accordo Culturale" firmato durante la visita ufficiale del Presidente della Repubblica Giovanni Gronchi nel Brasile di Juscelino Kubitschek (1958) sancì, come è noto, il definitivo superamento delle questioni pendenti.

Col passare degli anni, nella seconda metà del secolo scorso, questa temperie s'́ andò affievolendo, lasciando spazio a un approccio più marcatamente economicistico.

La ripresa di attenzione per le problematiche culturali e gli aspetti antropologici dei rapporti fra i due Paesi sembra essere avvenuta in concomitanza con le celebrazioni del quinto centenario della scoperta del Brasile, evento al quale le istituzioni italiane parteciparono in maniera significativa. La visita dell'allora Presidente della Repubblica, Carlo Azeglio Ciampi, ebbe un alto valore simbolico, al quale contribuì il conferimento di 
onorificienze ad autori e protagonisti della telenovela Terra Nostra, che raccontava la saga della grande emigrazione di fine Ottocento.

Da quel momento in poi la diplomazia ha valorizzato sempre più questo tipo di tematiche nello sviluppo delle sue strategie. Non è per caso che,come capodella legazione in Brasile, nel 2008 il Ministero degli Affari Esteri ha scelto Gherardo La Francesca, il diplomatico che in quel momento era Direttore Generale per la Promozione e la Cooperazione Culturale.Nella sua nuova, più delicata funzione egli ha messo in pratica $\mathrm{i}$ principi ai quali s'era ispirato in quella precedente, adattandoli alla situazione specifica con equilibrio e ampia visione strategica. La scelta del logotipo del "Momento Italia Brasile", realizzato dall'oriundo Washington Olivetto, rivela plasticamente il senso della missione di La Francesca. Nell'immagine stilizzata del Colosseo sormontato dal Cristo Corcovado sono riassunti secoli di una storia di amicizia, fratellanza, valori e interessi comuni. Di questa tradizione egli sè fatto interprete e protagonista, con l'ausilio di uno staff ugualmente motivato.

A conclusione della sua missione, l'ambasciatore è stato insignito dalla “Universidade do Estado do Rio de Janeiro“" del titolo di "Grão-Oficial da Ordem do Mérito José Bonifácio“; la più elevata onorificenza accademica gli è stata consegnata solennemente dal rettore, prof. Ricardo Vieiralves. In una sorta di vichiano ricorso storico, si potrebbe immaginare che, mediante questa cerimonia,il"Patriarca dell'Indipendenza del Brasile", l'arcade Américo Elísio, ha ritrovato Romanella figura dell'ambasciatore d'Italia. Il titolo concesso a La Francesca è un omaggio al Paeseda lui rappresentato ed alla grande comunità di oriundi che vivono in Brasile. Anche in questo modo si riconosce l'importanza della diplomazia nella costruzione di una nuova geopolitica, capace diesprimere i sentimenti di fratellanza "di due popoli, due culture, tanto distanziate fra loro nello spazio e, tuttavia, tanto vicine nelle loro radici comuni e secolari” (BUARQUE DE HOLANDA, 2002, p.108). 


\section{Referências}

AVELLA, Aniello Angelo. "Cinquecento Anni di Relazioni fra Brasile e Italia. Appunti per un metaviaggio nella Storia e nell'Invenzione Artistica“. Bérénice, n. 23, pp. 21-26.

----------. “A Itália e o Quinto Centenário do Descobrimento do Brasil”. Revista do Instituto Histórico e Geográfico Brasileiro, n. 420, 2003, pp. 225-231.

----------. “A Itália dos Brasileiros: História, Mito, Criação Literária”. Revista do Instituto Histórico e $\quad$ Geográfico Brasileiro, n. 425, 2004, pp. 95-105.

---------. Una Napoletana Imperatrice ai Tropici. Teresa Cristina di Borbone sul Trono del Brasile(1843-1889). Roma: Exòrma, 2012.

CASCUDO, Luís da Câmara. Em memória de Stradelli 1852-1926. $2^{\text {a }}$ ed. atualizada. Manaus: Edições do Governo do Estado do Amazonas, 1967 (1ª ed. 1936).

CENNI, Franco. Italianos no Brasil. São Paulo: Martins, 1960.

CERRETI, Claudio. Della Società Geografica Italiana e della sua Vicenda Storica (1867-1997). Roma: Società Geografica Italiana, 2000.

DE BONI, Luis A. (org.). A Presença Italiana no Brasil. Porto Alegre: EST, 1987. 2 vols.

HOLANDA, Sergio Buarque de. Apporto Italiano nella Formazione del Brasile/A Contribuição Italiana para a Formação do Brasil (ed. bilíngue). Florianópolis: Universidade Federal de Santa Catarina, $2002\left(1^{\mathrm{a}}\right.$. Ed. 1954).

JACOBELLI, Luciana. “L’UnicitàArcheologicadelTerritorio Vesuviano/A Unicidade Arqueológica do Território Vesuviano“. In JACOBELLI, Luciana (org.). Além de Pompeia. Redescobrindo os Encantos de Stabiae (ed. bilíngue). Rio de Janeiro: Editora Comunità, 2012. 
LA FRANCESCA, Gherardo. "Presentazione". In GIUDICE, Claudia (org.). Momento Itália/Brasil 2011/2012. Rio de Janeiro: Abril, 2012.

MARQUES, Adílio Jorge. O Professor do Jovem Imperador. Um Naturalista Luso-Brasileiro: Alexandre António Vandelli(1784-1862). Rio de Janeiro: Vieira \& Lent, 2010.

MEARSHEIMER, John J. The Tragedy of Great Power Politics. W. W. Norton \& Company: New York, 2001.

RIBEIRO, Darcy. "Siamo noi i neo-romani”. In Avella, A.A. (org.), Dal "Pan di Zucchero al Colosseo. Intellettuali Brasiliani a Spasso per leVie di Roma e Dintorni. Roma-L’Aquila: Japadre, 2006.

STRADELLI, Ermanno. "Vocabulários da língua geral portuguez-nheengatu e nheengatu-portuguez, precedidos de um esboço de grammatica nheengatu-umbue-sauamiri e seguidos de contos em língua geral". Revista do Instituto Histórico Geográfico Brasileiro, Rio de Janeiro, t. 104, vol 158, pp. 5-678, 1929.

STRADELLI, Ermanno. Lendas e Notas de Viagem. São Paulo: Martins Fontes, 2009.

VANNI, Julio Cezar. Italianos no Rio de Janeiro. Rio de Janeiro: Editora Comunità, 2000.

Artigo encaminhado para publicação em dezembro de 2012.

Artigo aceito para publicação em dezembro de 2012.

Geo UERJ - Ano 14, nº. 23,v. 2, $2^{\circ}$ semestre de 2012 p. 375-388

ISSN: 1415-7543 E-ISSN: 1981-9021

http://www.e-publicacoes.uerj.br/index.php/geouerj 